\title{
ROBUST AUDIO PRECOMPENSATION WITH PROBABILISTIC MODELING OF TRANSFER FUNCTION VARIABILITY
}

\author{
Lars-Johan Brännmark \\ Uppsala University \\ Department of of Engineering Sciences, Signals and Systems \\ PO Box 534, SE-751 21 Uppsala, Sweden
}

\begin{abstract}
A new approach to the single-channel loudspeaker equalization problem is presented. A scalar discrete-time mixed-phase precompensation filter is designed to be spatially robust, meaning that equalization performance should be insensitive to listener movements within a predefined spatial region. The problem is posed in a single-input multiple-output (SIMO) feedforward control framework and a polynomial solution is derived, based on a set of room transfer functions (RTFs) measured at a number of control points in the region, and a multipoint mean-square error (MSE) criterion. Spatial robustness is obtained by the introduction of two novel strategies. Firstly, a probabilistic model is used to describe the RTF variability around each control point, and the MSE criterion is averaged with respect to this variability. Secondly, the pre-response errors, normally associated with mixed-phase equalizer design, are alleviated by restricting the compensator to have a certain structure. The proposed method is shown to produce filters with excellent time- and frequency-domain performance.
\end{abstract}

Index Terms - Loudspeaker equalization, Acoustic signal processing, Robustness, Feedforward control, Pre-ringing

\section{INTRODUCTION}

In the design of single-channel loudspeaker equalization filters, spatial robustness is essential if the filters are to be practically useful. This aspect of equalizer design has been studied for many years, and several methods for robust filter design have been proposed, see e.g., [1-4]. A problem closely related to spatial robustness is that of pre-response errors, or pre-ringings, which occur in mixed-phase designs $[3,4]$. In general, an unconstrained mixed-phase filter design reduces the total MSE at the price of introducing pre-response errors, which are subjectively worse than causal, or post-response, errors. Traditionally, excessive pre- and post-ringing problems have been treated either by a restriction of the filter order, or by regularization techniques $[5,6]$. Pre-ringings can also be completely avoided by restricting the filter to be minimum phase, but then the time domain aspect of equalization is neglected.

In [4], the equalization problem was formulated in a singleinput multiple-output (SIMO) feedforward control setting, and it was shown that an optimal mixed-phase compensator with a very low level of pre-ringing can be obtained, if the compensator is constrained to have a certain structure. However, unless the number of measured RTFs is very large, this kind of design suffers from a lack of spatial information, and it was concluded in [4] that some amount of frequency response smoothing (e.g., 1/6th octave) is necessary

The author is also with Dirac Research AB, Hansellisgatan 6, SE-754 50 Uppsala, Sweden when computing the inverse filter, to avoid over-fitting of the filter to the chosen control points.

In this paper, we propose an extension of the SIMO feedforward approach. A probabilistic model called the extended design model [7] is introduced, to capture the unmeasured RTF variability between the control points. A robust controller is then obtained by minimization of an averaged multipoint MSE criterion, where the average is taken with respect to the random variables in the extended design model. The method is finally evaluated using measured data in a real listening environment.

Remarks on the notation: Filters and RTFs are represented by polynomials and rational functions in the backward timeshift operator $q^{-1},\left(q^{-1} s(k)=s(k-1)\right)$, where $q^{-1}$ corresponds to $z^{-1}$ in the frequency domain. A polynomial (polynomial matrix) is denoted by italic capital (bold capital) letters as $P\left(q^{-1}\right)=\mathrm{p}_{0}+\mathrm{p}_{1} q^{-1}+\cdots+\mathrm{p}_{n_{P}} q^{-n_{P}} \quad\left(\boldsymbol{P}\left(q^{-1}\right)=\right.$ $\left.\mathbf{P}_{0}+\mathbf{P}_{1} q^{-1}+\cdots+\mathbf{P}_{n_{\boldsymbol{P}}} q^{-n_{\boldsymbol{P}}}\right)$, whereas rational matrices, indicated by bold calligraphic letters, are represented on common denominator form as $\mathcal{G}\left(q^{-1}\right)=\boldsymbol{Q}\left(q^{-1}\right) / P\left(q^{-1}\right)$. Scalar rational functions are denoted by normal calligraphic letters as $\mathcal{G}\left(q^{-1}\right)$. For any polynomial (polynomial matrix) we define the conjugate as $P_{*}(q)=P(q)=\mathrm{p}_{0}+\mathrm{p}_{1} q+\cdots+\mathrm{p}_{n_{P}} q^{n_{P}}$ $\left(\boldsymbol{P}_{*}(q)=\boldsymbol{P}^{T}(q)=\mathbf{P}_{0}^{T}+\mathbf{P}_{1}^{T} q+\cdots+\mathbf{P}_{n_{P}}^{T} q^{n_{\boldsymbol{P}}}\right)$. The reciprocal $\bar{P}$ of a polynomial $P$ is defined as $\bar{P}\left(q^{-1}\right)=q^{-n_{P}} P_{*}(q)$ and the identity matrix of dimension $p$ is denoted $\mathbf{I}_{p}$. The arguments $q^{-1}, q$, $z^{-1}, z$ etc. are often omitted, unless there is a risk for confusion.

\section{PROBLEM DESCRIPTION}

\subsection{Probabilistic Modeling of RTF Variability}

In the present work, we shall use a so called extended design model to describe the assumed system behaviour throughout the listening region, based on information provided by a set of scalar RTFs acquired at $p$ control points. This model was introduced in [7] as a method for dealing with modeling errors in polynomial-based filtering and control problems. In brief, it can be described as follows. A multivariable rational transfer function is represented as

$$
\mathcal{H}\left(q^{-1}\right)=\mathcal{H}_{0}\left(q^{-1}\right)+\Delta \mathcal{H}\left(q^{-1}\right)
$$

where $\mathcal{H}_{0}$ represents a nominal model, and $\Delta \mathcal{H}$ is parameterized by zero-mean random variables and describes possible deviations around the nominal model. As an example, consider a scalar finite impulse response (FIR) model $B\left(q^{-1}\right)$ :

$$
\begin{aligned}
B\left(q^{-1}\right) & =b_{0}+b_{1} q^{-1}=b_{00}+b_{01} q^{-1}+\Delta b_{1} q^{-1} \\
& =B_{0}\left(q^{-1}\right)+\Delta B\left(q^{-1}\right)
\end{aligned}
$$


where $b_{0}=b_{00}$ is perfectly known, and uncertainty about $b_{1}$ is modeled with a zero-mean random variable $\Delta b_{1}$ having variance $\sigma_{\Delta b_{1}}^{2}$. In this framework, a robust estimator or feedforward controller is obtained by minimizing the averaged mean square error criterion

$$
J=\overline{\mathrm{E}} \mathrm{E}\left\{\operatorname{tr}\left(\varepsilon(k) \varepsilon^{T}(k)\right)\right\}
$$

where $\varepsilon(k)$ is a vector-valued error signal. In the criterion (3), $\mathrm{E}\{\cdot\}$ denotes expectation with respect to statistical properties of signals, whereas $\overline{\mathrm{E}}\{\cdot\}$ represents an expectation (marginalization) with respect to the random variables in $\Delta \mathcal{H}$, which are assumed to be independent of all involved signals. Here, this framework is applied to the acoustic SIMO model, using the following observations:

- The first 1-2 milliseconds of a room impulse response generally contains only the direct sound from the source. This part of the impulse is independent of the room environment, and is also rather insensitive to receiver position.

- If the wavelength is considerably larger than the distance between the control points, then the sound pressure between the control points can be almost linearly interpolated from the pressure at the control points.

- At low frequencies, in particular below the Schroeder frequency [8], the sound field in a room is less diffuse than at high frequencies.

- At high frequencies, the reverberant part of the impulse response (arriving a few milliseconds after the direct sound) is diffuse, and varies unpredictably between the control points.

Based on these prior physical insights, a relevant extended design model of the acoustic SIMO system can be obtained by letting the nominal model $\mathcal{H}_{0}$ contain the direct sound and the low-frequency parts of the RTFs, while the stochastic part $\Delta \mathcal{H}$ models the reverberant, "random" high-frequency part. This partitioning of the RTFs is accomplished by filtering out the high-frequency reverberant part from each measured impulse response. The filtered-out reverberant parts are then used for constructing the set of models $\Delta \mathcal{H}$. In more detail, the RTF at the $i$ th control point, denoted $\mathcal{H}_{i}$, is expressed as

$$
\mathcal{H}_{i}=\mathcal{H}_{0 i}+\Delta \mathcal{H}_{i}=\mathcal{H}_{0 i}+\frac{\Delta B_{i} B_{1}}{A_{1}}
$$

The coefficients of the polynomial (FIR filter) $\Delta B_{i}$ are assumed to be be independent and identically distributed (i.i.d.) random variables which are normalized so that $\overline{\mathrm{E}}\left\{\Delta B_{i *} \Delta B_{i}\right\}=1 . B_{1} / A_{1}$ is a "shaping filter" which, to some relevant level of detail, models the spectral envelope of the reverberant sound. We have chosen to use a common shaping filter $B_{1} / A_{1}$ for all outputs in the SIMO model, because we have no reason to believe that a loudspeaker's reverberant field differs significantly in a statistical sense between separate control points. We also assume that the random parts $\Delta B_{i}$ are uncorrelated between different outputs, i.e., $\overline{\mathrm{E}}\left\{\Delta B_{i *} \Delta B_{j}\right\}=0$ if $i \neq j$. The order of the random polynomials $\Delta B_{i}$ is so far unspecified.

\subsection{Robust Feedforward Control With Pre-Ringing Constraint}

Let the multi-point error signal of a compensated SIMO acoustical system, with scalar input $w(k)$, be described by

$$
\boldsymbol{y}(k)=\mathcal{D} w(k)-\mathcal{H} \mathcal{R} w(k)
$$

where $\mathcal{H}\left(q^{-1}\right)$ and $\mathcal{D}\left(q^{-1}\right)$ are the RTFs and the desired responses, respectively, between the loudspeaker and $p$ control points. $\mathcal{H}\left(q^{-1}\right)$ and $\mathcal{D}\left(q^{-1}\right)$ are represented on right matrix fraction description form [9] as

$$
\begin{aligned}
\mathcal{H} & =\mathcal{H}_{0}+\Delta \mathcal{H}=\left[\begin{array}{c}
B_{01} \\
\vdots \\
B_{0 p}
\end{array}\right] \frac{1}{A_{0}}+\left[\begin{array}{c}
\Delta B_{1} \\
\vdots \\
\Delta B_{p}
\end{array}\right] \frac{B_{1}}{A_{1}} \\
& =\boldsymbol{B}_{0} \frac{1}{A_{0}}+\Delta \boldsymbol{B} B_{1} \frac{1}{A_{1}}=\left(\boldsymbol{B}_{0} A_{1}+\Delta \boldsymbol{B} B_{1} A_{0}\right) \frac{1}{A_{0} A_{1}} \\
& =\left(\hat{\boldsymbol{B}}_{0}+\Delta \boldsymbol{B} \hat{B}_{1}\right) \frac{1}{A}=\boldsymbol{B} / A \\
\mathcal{D} & =\left[\begin{array}{c}
D_{1} \\
\vdots \\
D_{p}
\end{array}\right] \frac{1}{E}=\boldsymbol{D} / E,
\end{aligned}
$$

where $\boldsymbol{B}=\hat{\boldsymbol{B}}_{0}+\Delta \boldsymbol{B} \hat{B}_{1}, \hat{\boldsymbol{B}}_{0}=\boldsymbol{B}_{0} A_{1}, \hat{B}_{1}=B_{1} A_{0}$ and $A=A_{0} A_{1}$. Here, $A$ and $E$ are stable and monic polynomials, and $\mathcal{R}$ is a stable compensator operating on the source signal $w(k)$. To obtain optimum performance without introducing pre-ringing errors, the compensator is restricted to have the form $\mathcal{R}\left(q^{-1}, q\right)=$ $q^{-d} \mathcal{F}_{*}(q) \mathcal{R}_{1}\left(q^{-1}\right)$, where $\mathcal{F}\left(q^{-1}\right)=\bar{F}\left(q^{-1}\right) / F\left(q^{-1}\right)$ is an assumed common excess phase part of all RTFs in the region, $d$ is the smallest power of $q^{-1}$ occurring in any of the desired responses in $\mathcal{D}\left(q^{-1}\right)$, and $\mathcal{R}_{1}\left(q^{-1}\right)$ is an arbitrary stable and causal filter, see [4] for details. Finally we introduce the weighted error signal $\boldsymbol{z}_{1}(k)=\boldsymbol{V} \boldsymbol{y}(k)$ and the weighted control effort signal $z_{2}(k)=W u(k)$, where $\boldsymbol{V}$ is a diagonal polynomial matrix of dimension $p \mid p$, and $W$ is a scalar polynomial. The structure of this problem is illustrated in Fig. 1. The robust SIMO controller is

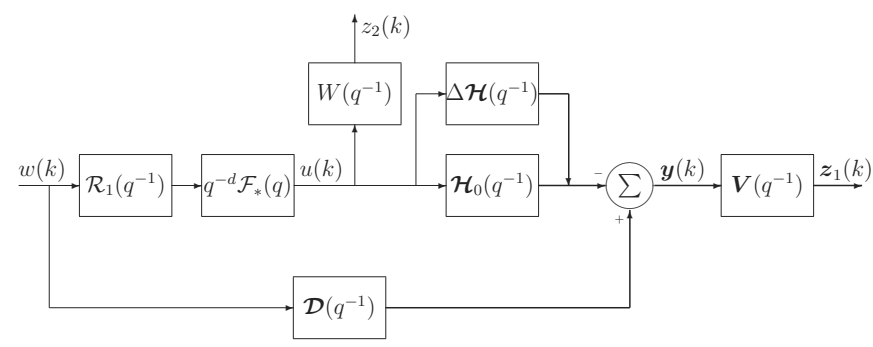

Fig. 1. Block diagram of the robust SIMO feedforward control problem. The thin lines represent scalar signals, and the thick lines represent vector-valued signals of dimension $p$.

obtained by finding the causal filter $\mathcal{R}_{1}$ that minimizes the averaged MSE criterion

$$
\begin{aligned}
J & =\overline{\mathrm{E}} \mathrm{E}\left\{\operatorname{tr}\left(\left[\begin{array}{l}
\boldsymbol{z}_{1} \\
z_{2}
\end{array}\right]\left[\begin{array}{ll}
\boldsymbol{z}_{1}^{T} & z_{2}^{T}
\end{array}\right]\right)\right\} \\
& =\overline{\mathrm{E}} \mathrm{E}\left\{\operatorname{tr}\left(\boldsymbol{V} \boldsymbol{y}(k)(\boldsymbol{V} \boldsymbol{y}(k))^{T}\right)+\operatorname{tr}\left(W u(k)(W u(k))^{T}\right)\right\} .
\end{aligned}
$$

Note that in practice the compensator $\mathcal{R}$ must be causal, and this can be accomplished by replacing the noncausally decaying all-pass filter $\mathcal{F}_{*}(q)$ with an FIR approximation $\mathcal{F}_{*}^{\text {FIR }}(q)$ of order $d$, so that $q^{-d} \mathcal{F}_{*}^{\text {FIR }}(q)$ becomes causal. If $d$ is chosen large enough, this approximation has negligible influence on the final result.

\subsection{The Robust Optimal Controller}

Using the methods developed in [10], it can be shown that the optimal causal compensator $\mathcal{R}_{1}\left(q^{-1}\right)$ that minimizes (8) is given by

$$
\mathcal{R}_{1}=\frac{Q A}{\beta E F}
$$


where $\beta\left(q^{-1}\right)$ is obtained from the averaged spectral factorization

$$
\begin{aligned}
& \beta_{*} \beta=\overline{\mathrm{E}}\left\{\boldsymbol{B}_{*} \boldsymbol{V}_{*} \boldsymbol{V} \boldsymbol{B}+A_{*} W_{*} W A\right\} \\
& \quad=\hat{\boldsymbol{B}}_{0 *} \boldsymbol{V}_{*} \boldsymbol{V} \hat{\boldsymbol{B}}_{0}+\hat{B}_{1 *} \overline{\mathrm{E}}\left\{\Delta \boldsymbol{B}_{*} \boldsymbol{V}_{*} \boldsymbol{V} \Delta \boldsymbol{B}\right\} \hat{B}_{1}+A_{*} W_{*} W A \\
& =\hat{\boldsymbol{B}}_{0 *} \boldsymbol{V}_{*} \boldsymbol{V} \hat{\boldsymbol{B}}_{0}+\hat{B}_{1 *} \operatorname{tr}\left(\overline{\mathrm{E}}\left\{\Delta \boldsymbol{B} \Delta \boldsymbol{B}_{*}\right\} \boldsymbol{V}_{*} \boldsymbol{V}\right) \hat{B}_{1}+A_{*} W_{*} W A
\end{aligned}
$$

and $Q\left(q^{-1}\right)$, along with a polynomial $L_{*}(q)$, constitute the unique solution to the polynomial Diophantine equation

$$
\hat{\boldsymbol{B}}_{0 *} \boldsymbol{V}_{*} \boldsymbol{V} \boldsymbol{D} \bar{F} q^{d}=\beta_{*} Q+L_{*} z F E .
$$

Using (9)-(11), the total compensator $\mathcal{R}=q^{-d} \mathcal{F}_{*} \mathcal{R}_{1}$ can be expressed on the form

$$
\mathcal{R}=q^{-d} \mathcal{F}_{*}\left\{\frac{\hat{\boldsymbol{B}}_{0 *} \boldsymbol{V}_{*} \boldsymbol{V} \boldsymbol{D}}{\beta_{*} E} \mathcal{F} q^{d}\right\}_{+} \frac{A}{\beta}
$$

where the operator $\{\cdot\}_{+}$yields the causal part of the function within the brackets. Note that, since we here assume that the coefficients of $\Delta B_{i}, i \in\{1, \ldots, p\}$ are all i.i.d. random variables, we have that $\overline{\mathrm{E}}\left\{\Delta \boldsymbol{B} \Delta \boldsymbol{B}_{*}\right\}=\mathbf{I}_{p}$. If furthermore no frequency dependent weighting $\boldsymbol{V}$ is applied to the error signal $\boldsymbol{y}(k)$ in (8), then also $\boldsymbol{V}_{*} \boldsymbol{V}=\mathbf{I}_{p}$ and $\operatorname{tr}\left(\overline{\mathrm{E}}\left\{\Delta \boldsymbol{B} \Delta \boldsymbol{B}_{*}\right\} \boldsymbol{V}_{*} \boldsymbol{V}\right)=p$, in which case (10)-(12) are greatly simplified.

\section{A DESIGN EXAMPLE}

In this section, the above theory is applied to a practical filter design case, using measured impulse responses in a room. We begin with an overview of necessary structural choices and parameter settings.

\subsection{Modeling Considerations}

In the following example, we shall use high-order FIR models as RTFs in $\mathcal{H}_{0}$, i.e., $\mathcal{H}_{0 i}=B_{0 i}$ and $A_{0}=1$. These models are obtained by applying a variable low-pass filter to each of the $p$ measured impulse responses, yielding nominal responses $B_{0 i}$ and their corresponding "random" reverberant parts $B_{r i}$. The cutoff frequency $f_{C}$ of the variable filter decreases from $22050 \mathrm{~Hz}$ to $300 \mathrm{~Hz}$ over a time frame of $6 \mathrm{~ms}$, starting at $0.8 \mathrm{~ms}$ after the first peak in the impulse response. This process is illustrated in Fig. 2. The shaping filter $B_{1} / A_{1}$, intended to model the spectral

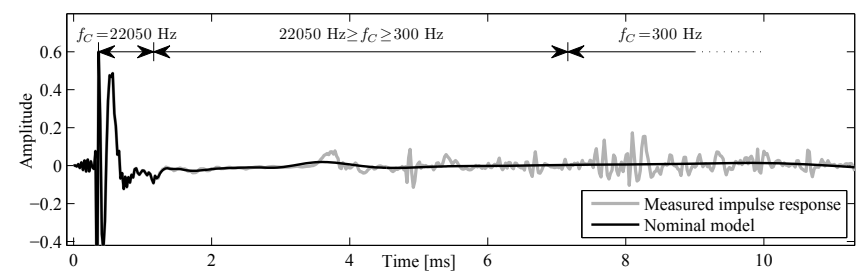

Fig. 2. To obtain a nominal model $B_{0 i}$ (black line), a measured impulse response (grey line) is low-pass filtered using a cutoff frequency $f_{C}$ that decreases with time.

envelope of the reverberant sound, is chosen to be an FIR filter of order 300 , and thus $A_{1}=1$. The FIR shaping filter $B_{1}$ is constructed from the reverberant parts $B_{r i}$ by using their average periodogram $\Phi_{\mathrm{av}}=\frac{1}{p} \sum_{1}^{p} B_{r i *} B_{r i}$. A triangular window of length 599 is applied symmetrically over the polynomial coefficients of $\Phi_{\text {av }}$, yielding an averaged Blackman-Tukey spectral estimate $\hat{\Phi}_{\text {av }}$ of the reverberant sound. The shaping filter $B_{1}$ is finally obtained as the minimum phase spectral factor of $\hat{\Phi}_{\mathrm{av}}$. The target dynamics $\mathcal{D}_{i}$ are here chosen to be "ideal", i.e., pure delays, so that $E=1$ and
$\mathcal{D}_{i}=D_{i}=q^{-\left(\Delta_{i}+d\right)}$, where $\Delta_{i}$ is the acoustic propagation delay between the loudspeaker and the $i$ th control point and $d$ is a common additional delay of 8820 samples $(0.2 \mathrm{sec}$ at $44100 \mathrm{~Hz}$ sampling frequency). The control effort weighting polynomial $W$ is designed to penalize excessive gains below $40 \mathrm{~Hz}$ and above $21000 \mathrm{~Hz}$. The error signal weighting matrix is not used, i.e., $\boldsymbol{V}=\mathbf{I}_{p}$. With the above parameterizations, the filter becomes

$$
\mathcal{R}\left(q^{-1}, q\right)=q^{-d} \frac{\bar{F}_{*}(q)}{F_{*}(q)} \frac{Q\left(q^{-1}\right)}{\beta\left(q^{-1}\right) F\left(q^{-1}\right)}
$$

where $\beta$ and $Q$ are given by the spectral factorization

$$
\beta_{*} \beta=\boldsymbol{B}_{0 *} \boldsymbol{B}_{0}+p B_{1 *} B_{1}+W_{*} W
$$

and the Diophantine equation

$$
\boldsymbol{B}_{0 *} \boldsymbol{D} \bar{F} q^{d}=\beta_{*} Q+L_{*} z F
$$

The filter $\mathcal{F}_{*}=\bar{F}_{*} / F_{*}$ is constructed by detection of excess phase zero clusters, as described in [4].

\subsection{Experimental Conditions}

In a room of dimensions $4.5 \times 6 \times 2.6 \mathrm{~m}$ and with a loudspeakermicrophone distance of about $2.5 \mathrm{~m}$, RTF measurements were performed at altogether 18 control points, randomly chosen within a volume of $35 \times 35 \times 35 \mathrm{~cm}$. The duration of the so obtained impulse responses was 0.4 seconds or 18000 samples. Of these 18 responses, 9 were used for filter design, and 9 were saved for validation purposes. Thus $p=9$, and the systems $\mathcal{H}, \mathcal{D}, \boldsymbol{B}_{0}, \Delta \boldsymbol{B}, \boldsymbol{D}$ etc. in (6)-(7) are polynomial matrices of dimension $9 \mid 1$. A causal version of $\mathcal{R}$ was computed according to (13)-(15), using the FIR approximation of $\mathcal{F}_{*}$ as described in subsection 2.2.

\subsection{Results}

To graphically assess the qualities of the suggested filter design, we shall use the following tools: 1) the average frequency response, 2) the impulse response maximum level envelope, 3) the average energy step response below $320 \mathrm{~Hz}$, and 4) the average Schroeder decay sequence below $320 \mathrm{~Hz}$. (Average here refers to a spatial average over the responses, in either the design or validation points, see [4] for further details). We begin however by examining the spectral properties of the minimum-phase polynomial $\beta$, which greatly influences the magnitude response of the final filter $\mathcal{R}$. Fig. 3 shows the power response $\beta_{*} \beta$ and its constituent parts belonging, respectively, to the nominal models $\boldsymbol{B}_{0}$, the spectral envelope $B_{1}$ of the reverberant model, and the control signal penalty $W$. One can con-

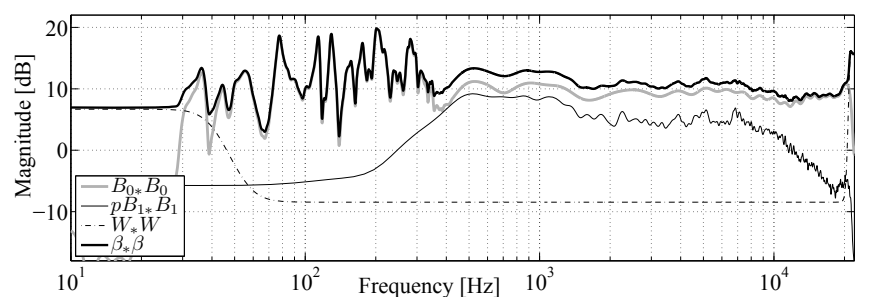

Fig. 3. Frequency responses of $\beta_{*} \beta$ and its components $\boldsymbol{B}_{0 *} \boldsymbol{B}_{0}$, $p B_{1 *} B_{1}$ and $W_{*} W$, as given by (14).

clude from Fig. 3 that the reflection filtering above $300 \mathrm{~Hz}$, together with the statistical model $\Delta \boldsymbol{B} B_{1}$ of the reverberant parts, clearly has a smoothing effect on $\beta$ above $300 \mathrm{~Hz}$, while below $300 \mathrm{~Hz}, \beta$ 


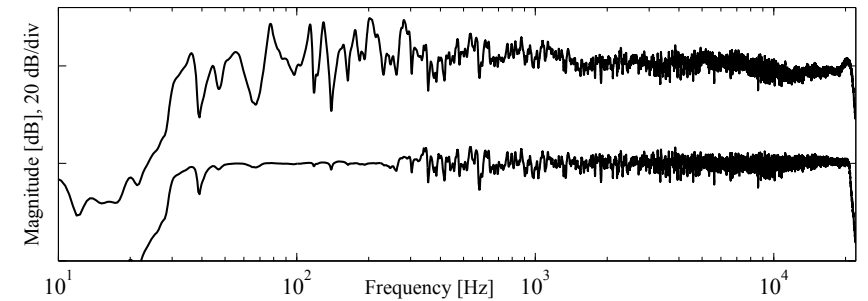

(a)

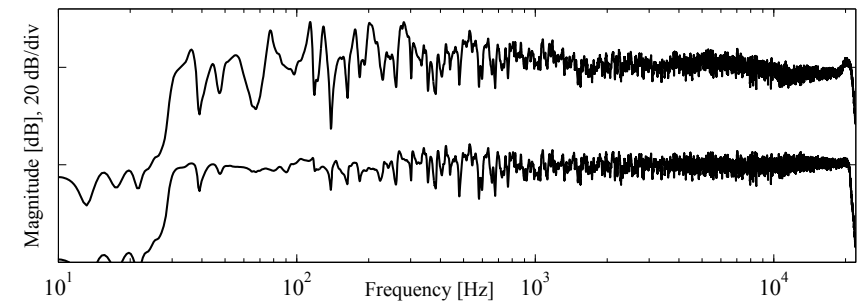

(b)

Fig. 4. Average frequency responses of original (top) and equalized (bottom) system. (a) Design points. (b) Validation points.

is very detailed. Below $30 \mathrm{~Hz}$ and above $21 \mathrm{kHz}, \beta$ is completely determined by $W$. In Fig. 4, one sees that the filter achieves a nearly equal amount of spectral flattening in both the design and validation points. Fig. 5 shows the maximum absolute value of 9 time-aligned and normalized responses, clearly showing a low pre-ringing level, as well as an improved peak-to-tail ratio, both in the design and validation points. Finally, in Fig. 6, the filter is seen to considerably

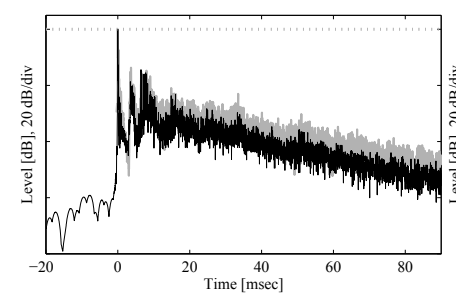

(a)

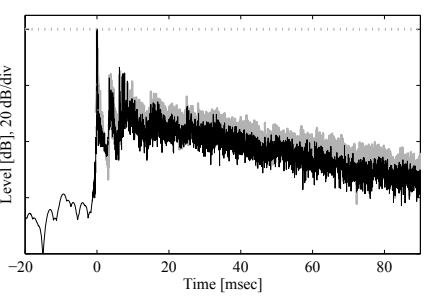

(b)
Fig. 5. Maximum level envelopes of original (grey) and equalized (black) impulse responses. (a) Design points. (b) Validation points.

improve the time domain performance at low frequencies, by shortening the energy rise time of the system, or equivalently as shown in Fig. 7, by shortening the initial energy decay. Summing up the above assessment, the filter clearly improves the system performance according to all of the examined properties, and the improvement is nearly equal in both the design and validation points. Thus, the suggested design is successful in obtaining filters which are spatially robust without being overly conservative.

\section{CONCLUSIONS AND FUTURE RESEARCH}

A novel framework for spatially robust single-channel audio compensation has been presented. Using a concept from polynomialbased robust filtering and control, a spatially robust filter was designed and evaluated, using measurements in a real room. The filter was verified to considerably improve system performance within the chosen listening region in a spatially robust way. The proposed framework is however open to further refinement, for example by exploiting more prior acoustical knowledge in the nominal/stochastic partitioning of $\mathcal{H}=\mathcal{H}_{0}+\Delta \mathcal{H}$. Firstly, the nonstationary reflection filtering operation that was used in the design of $\mathcal{H}_{0}$ could be

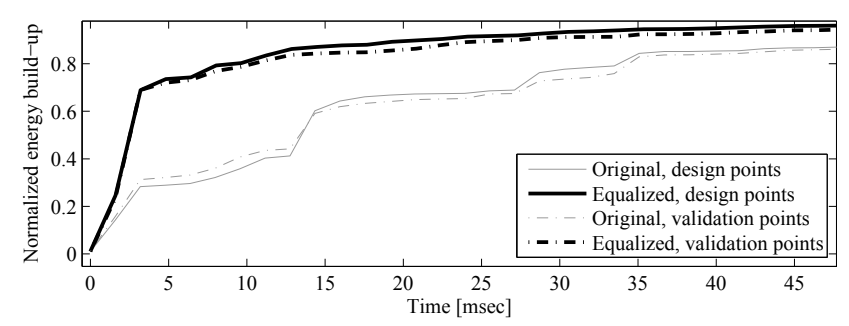

Fig. 6. Average energy step responses below $320 \mathrm{~Hz}$ of original system (black) and equalized system (grey), in the design points (solid) and in the validation points (dash-dotted lines).

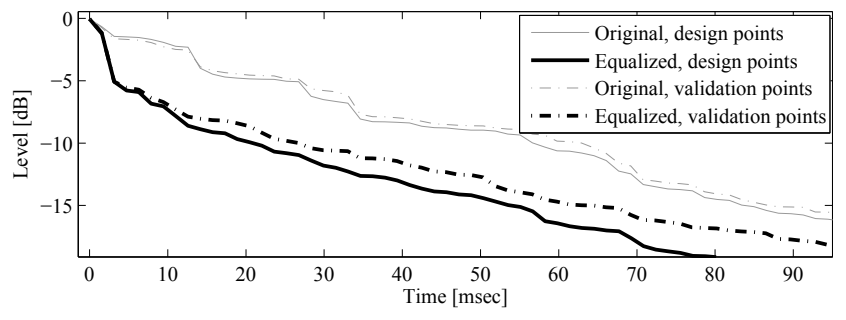

Fig. 7. Average Schroeder decay sequences below $320 \mathrm{~Hz}$ of original system (black) and equalized system (grey), in the design points (solid) and in the validation points (dash-dotted lines).

more carefully designed, and secondly, spatial dependencies (crosscorrelations) among the stochastic coefficients in $\Delta \boldsymbol{B}$ could be used in the extended design model. The probabilistic framework for RTF modeling is also expected to be useful in acoustic MIMO applications.

\section{REFERENCES}

[1] S. J. Elliott and P. A. Nelson, "Multiple-point equalization in a room using adaptive digital filters," J. Audio Eng. Soc., vol. 37, no. 11, pp. 899-907, November 1989.

[2] R. Wilson, "Equalization of loudspeaker drive units considering both on- and off-axis responses," J. Audio Eng. Soc., vol. 39, no. 3, pp. 127-139, March 1991.

[3] P. Hatziantoniou and J. Mourjopoulos, "Errors in real-time room acoustics dereverberation,” J. Audio Eng. Soc., vol. 52, no. 9, pp. 883-899, September 2004.

[4] L.-J. Brännmark and A. Ahlén, "Spatially robust audio compensation based on SIMO feedforward control," IEEE Transactions on Signal Processing, vol. 57, no. 5, May 2009.

[5] O. Kirkeby and P. A. Nelson, "Digital filter design for inversion problems in sound reproduction," J. Audio Eng. Soc., vol. 47, no. 7/8, pp. 583-595, July/August 1999.

[6] O. Kirkeby, P. A. Nelson, H. Hamada, and F. Orduna-Bustamante, "Fast deconvolution of multichannel systems using regularization," IEEE Transactions on Speech and Audio Processing, vol. 6, no. 2, pp. 189194, March 1998.

[7] M. Sternad and A. Ahlén, "Robust filtering and feedforward control based on probabilistic descriptions of model errors," Automatica, vol. 29, no. 3, pp. 661-679, 1993.

[8] M. R. Schroeder, "Statistical parameters of the frequency response curves of large rooms," J. Audio Eng. Soc., vol. 35, no. 5, pp. 299-306, May 1987.

[9] T. Kailath, Linear Systems, Prentice-Hall, Englewood Cliffs, NJ.

[10] K. Öhrn, A. Ahlén, and M. Sternad, "A probabilistic approach to multivariable robust filtering and open-loop control," IEEE Transactions on Automatic Control, vol. 40, no. 3, pp. 405-418, March 1995. 